\title{
Plasmodium vivax cerebral malaria in an adult patient in Sudan
}

\author{
Maowia M. Mukhtar ${ }^{1 *}$, Omer A. Eisawi², Seth A. Amanfo ${ }^{3}$, Elwaleed M. Elamin' ${ }^{1}$ Zeinab S. Imam¹, \\ Faiza M. Osman ${ }^{4}$ and Manasik E. Hamed ${ }^{1}$
}

\begin{abstract}
Background: Plasmodium vivax infection is rising in sub-Saharan Africa, where Plasmodium falciparum is responsible for more than $90 \%$ of malaria cases. While P. vivax is identified as a major cause of severe and cerebral malaria in South east Asia, the Pacific and South America, most of the severe and cerebral cases in Africa were attributed to P. falciparum. Cases of severe malaria due to P. vivax are emerging in Africa. A few severe P. vivax cases were reported in Eastern Sudan and they were underestimated due to the lack of accurate diagnosis, low parasitaemia and seldom use of rapid diagnostic tests (RDTs).

Case presentation: A 60-year-old Sudanese male presented to the Al Kuwaiti hospital in the Sudan capital Khartoum. On admission, the patient was complaining of fever (measured temperature was $38^{\circ} \mathrm{C}$ ), sweating, chills, vomiting and confusion in the past 2 days prior to his admission. He rapidly deteriorated into a coma state within $48 \mathrm{~h}$ of the admission, with significant neck stiffness. He was admitted to the intensive care unit and was suspected of meningitis. Lumbar puncture was not performed since the patient was suffering from spinal cord disc. Brain CT scan was unremarkable. Several biochemical, haematological tests, and blood film for malaria were performed. The results of the laboratory tests were within the normal range except of mild elevation of the total white blood cell count and a significant decrease in the platelets count. Malaria parasites were seen in the blood film with high parasitaemia (quantified as $3+++$ ). The patient was diagnosed as P. vivax cerebral malaria based on the positive blood film and the amplification of $P$. vivax specific 499 bp amplicon using Plasmodium multi-species multiplex Polymerase Chain Reaction (PCR). The patient was treated with quinine $10 \mathrm{mg} / \mathrm{kg}$ body weight for 10 days followed by primaquine $15 \mathrm{mg} /$ days PO for 2 weeks. The symptoms subsided within $48 \mathrm{~h}$ and the patients was cured and released from the hospital.
\end{abstract}

Conclusions: Plasmodium vivax is an emerging cause of cerebral malaria in adults in Sudan and should be considered in the differential diagnosis of cerebral malaria for proper management of patients.

Keywords: Plasmodium vivax, Cerebral malaria, Sudan

\section{Background}

Plasmodium vivax infection is geographically widely spread. In 2013, it comprised $8 \%$ of all estimated malaria cases globally, and represented $47 \%$ of cases outside subSaharan Africa, and $<1 \%$ of cases in the WHO African Region [1]. According to the World Health Organization (WHO), there were about 15.8 million $P$. vivax cases in 2013, with the total number of malaria deaths that

\footnotetext{
*Correspondence: mmukhtar@tropmedicine.org

${ }^{1}$ Bioscience Research Institute, Ibn Sina University, P.O. Box 11463,

Khartoum, Sudan

Full list of author information is available at the end of the article
}

could be attributed to $P$. vivax ranging between 1900 and 10,000 globally.

Severe cases and deaths due to $P$. vivax malaria were reported from all endemic regions. In 2015, severe vivax malaria was attributed to cause $16 \%$ of all malaria related mortality outside sub-Saharan Africa [2]. The risk of death from $P$. vivax malaria was estimated ranging between 0.012 and $0.063 \%$, while the risk of severe disease was estimated between 0.29 and $0.82 \%$ [2].

Plasmodium vivax infection is an emerging public health problem in Sudan with an overall prevalence of $26.6 \%$ among malaria cases in different regions of the 
country [3]. Severe $P$. vivax cases were mostly neglected and misdiagnosed as Plasmodium falciparum infection or confused with other fever causing disease. There were a few reports of severe P. vivax malaria cases in Sudan, suggesting an underestimation of the prevalence of severe $P$. vivax infection due to the low sensitivity of the currently used blood films and the seldom use of rapid diagnostic tests (RDT) [4-6].

There is a need for accurate and rapid diagnosis of severe $P$. vivax infection for differential diagnosis form $P$. falciparum cerebral cases for effective management of patients.

\section{Case presentation}

A 60-year-old Sudanese male presented to the Al Kuwaiti hospital in the Sudan capital Khartoum complaining of fever (measured temperature was $38^{\circ} \mathrm{C}$ ) associated with sweating, chills, vomiting and confusion for 2 days prior to admission. He had been diagnosed with poorly managed diabetes mellitus for 20 years, and he was on Insulin Mixtard 30/70.

On his admission, the patient did not complain of any gastrointestinal Tract (GIT), urinary or respiratory disorders, but was very ill and feverish. Within $48 \mathrm{~h}$ post admission he rapidly deteriorated into a coma state; Glasgow coma scale of 6 and did not react to stimuli with significant neck stiffness. He was admitted to the intensive care unit and was suspected of meningitis. Lumbar puncture was not performed since the patient had spinal cord disc prolapse. Brain CT scan was unremarkable. Several biochemical and haematological tests and blood film for malaria were performed. The renal and liver parameters were within the normal range. The patient had a fasting blood sugar of $295 \mathrm{mg} / \mathrm{dl}$, haemoglobin of $11 \mathrm{~g} / \mathrm{dl}$, a platelet count of 43,000 cells $/ \mathrm{mm} 3$, erythrocyte sedimentation rate (ESR) $18 \mathrm{~mm}$, and a total white blood count of 13.4000 cells $/ \mathrm{mm}^{3}$.

Blood film for malaria parasites was positive by light microscopy for $P$. vivax (quantified as $3+++$ ). PCR amplified P. vivax specific band of 499 bp using Plasmodium multi species multiplex PCR primers (Fig. 1). The patient was diagnosed as $P$. vivax cerebral malaria case. He was started on intravenous fluids, intravenous quinine at the dose of $10 \mathrm{mg} / \mathrm{kg}$ body weight IV for 10 days and Ceftriaxone $1 \mathrm{~g}$ IV PD for 7 days, followed by primaquine $15 \mathrm{mg} /$ days $\mathrm{PO}$ for 2 weeks. The vital signs improved over the next $48 \mathrm{~h}$. The patient was released from the hospital 12 days post admission.

\section{Discussion and conclusions}

The incidence of $P$. vivax infection is rising in Sudan and has increased from about 5\% in 2013 to $26 \%$ of the total malaria cases [3]. Although severe P. vivax cases were

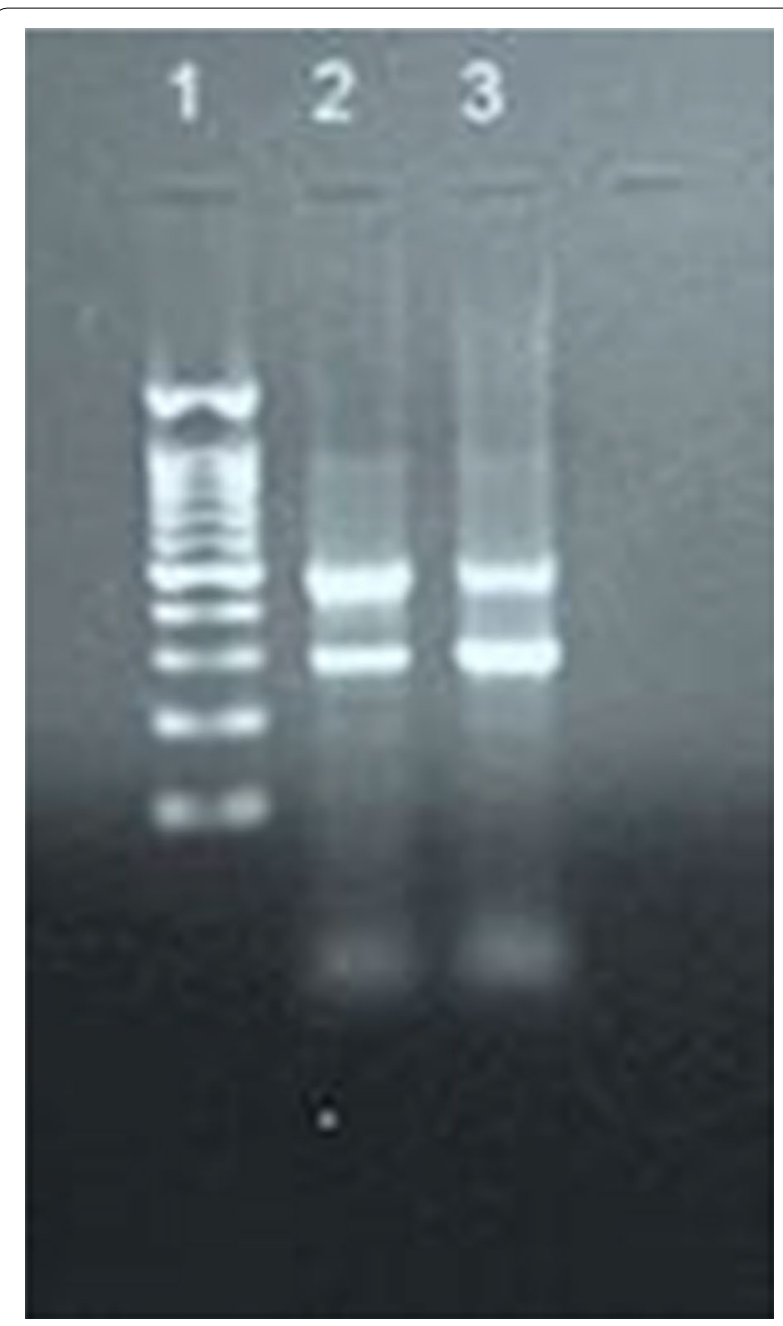

Fig. 1 Polymerase chain reaction amplification of Plasmodium vivax DNA of the blood sample from the patient showing amplification of 499 bp amplicon characteristic of P. vivax. Lane1: Molecular weight standard $100 \mathrm{bp}$, lane 2 positive $P$. vivax control, lane 3: patient's sample

reported from many endemic areas in South East Asia, the Pacific islands and South America, a few cases were reported in Africa reflecting the neglected status of $P$. vivax in Africa [2].

The patient described in the report was living in Khartoum the capital city of Sudan where malaria transmission is seasonal and non-endemic of $P$. vivax until recently. The fact that he was a known type 2 diabetic patients, presenting with high grade fever, chills, vomiting, sweating and confusion lead to suspicion of serious infections, including meningitis and falciparum malaria, which is the common cause of severe malaria in Sudan [5]. The rapid deterioration of the patient into coma and stiffness within $48 \mathrm{~h}$ of his admission increased the suspicion of meningitis and cerebral malaria and required 
his admission to the Intensive Care Unit (ICU). Cases of bacterial and viral meningitis were previously reported in Khartoum [6]. Lumbar puncture was recommended but the procedure was not performed since the patient had spinal cord disc making it a risky procedure. The negative finding of the CT scan excluded the possibility of central nervous system disease. The normal results of laboratory investigations including renal and liver parameters excluded renal and liver diseases. The lack of GIT and respiratory complain make the possibility of enteric infection and pulmonary TB less likely. On the other hand, the slight increase of the WBC suggested inflammatory reaction, while the low platelet counts increased the suspicion of severe infection including cerebral malaria. The finding of the blood film was decisive and demonstrated malaria parasite with high parasitaemia $(+++)$, close examination of the thin films identified P. vivax infection which was confirmed by the positive result of Plasmodium multi species PCR [7]. Interestingly, most of the severe $P$. vivax malaria reported in other endemic areas including those in children in eastern Sudan were accompanied by low parasitaemia contrary to the findings of this study [8]. The fact that the patient was a known type 2 diabetes patient might had impaired his immune response leading to the increase in the parasite replication. It is worth noting that, this patient had an attack of severe $P$. vivax malaria 3 months before his current illness. He presented with fever, chills and confusion. He was successfully treated with quinine $10 \mathrm{mg} / \mathrm{kg}$ body weight IV for 10 days, ceftazidime $1 \mathrm{G} \mathrm{IV}$ 8 hourly for 7 days followed by primaquine $15 \mathrm{mg} /$ days PO for 2 weeks evident by the negative blood film at the end of the treatment course. The recent attack could be a recurrent attack since $P$. vivax prevails at low parasitaemia making its detection difficult [9]. It is worth noting that no previous report on $P$. vivax drug resistance in Sudan, however the possibility of noncompliance to primaquine treatment could be excluded strengthening the possibility of the persistent of the parasite from the first infection [10].

This report identified $P$. vivax as a cause of cerebral malaria in adults in Sudan and recommends considering $P$. vivax in the differential diagnosis of cerebral malaria caused by $P$. falciparum that requires a different treatment regimen. Plasmodium vivax treatment should be closely supervised to assure cure of the infection.

\footnotetext{
Acknowledgements

We would like to thank Professor Mark Woolhouse and the secretariat of TIBA Consortium (Tackling Infections to Benefit Africa) at Edinburgh University for their support and the medical staff at the Alkuwaiti Specialized hospital for their support and excellent care during the hospital stay of the patient.
}

\section{Authors' contributions}

Professor Dr. MMM contributed to the design of the study, diagnosis of the case and writing of the report. Dr. OE contributed to the clinical management of the patient. SA contributed to the writing of the proposal. Design and writing of the manuscript. Professor EME co tribute to the laboratory diagnosis of the case. Dr. $\mathrm{ZI}$ contributed to the laboratory analysis of the samples. Dr. MEH contribute to the molecular analysis of the samples. Dr. FO contributed to the analysis of the samples and writing of the manuscript. All authors read and approved the final manuscript.

\section{Funding}

This research was commissioned by the National Institute for Health Research (NIHR) Global Health Research programme using UK aid from the UK Government. The views expressed in this publication are those of the author(s) and not necessarily those of the NIHR or the Department of Health and Social Care.

\section{Availability of data}

Not applicable.

\section{Ethics approval and consent to participate}

The study was approved by the national ethics committee at the Federal Ministry of Health, Sudan, part of the TIBA consortium project in Sudan.

\section{Consent for publication}

Written consent was obtained from the patient for publication.

\section{Competing interests}

The authors declare that they have no competing interests.

\section{Author details}

1 Bioscience Research Institute, Ibn Sina University, P.O. Box 11463, Khartoum, Sudan. ${ }^{2}$ Department of Medicine, Aliaa' Specialized Hospital, Khartoum, Sudan. ${ }^{3}$ NIHR Global Health Research Unit Tackling Infections to Benefit Africa at the University of Edinburgh, Usher Institute, University of Edinburgh, Edinburgh, UK. ${ }^{4}$ Institute of Endemic Diseases, University of Khartoum, Khartoum, Sudan.

Received: 28 May 2019 Accepted: 11 September 2019

Published online: 18 September 2019

\section{References}

1. World Health Organization. Severe malaria. Trop Med Int Health. 2014;19(Suppl 1):7-131.

2. WHO. Control and elimination of Plasmodium vivax malaria. A technical brief. Geneva: World Health Organization; 2015.

3. Elgoraish AG, Elzaki SEG, Ahmed RT, Ahmed Al, Fadlalmula HA, Abdalgader Mohamed $\mathrm{S}$, et al. Epidemiology and distribution of Plasmodium vivax malaria in Sudan. Trans R Soc Trop Med Hyg. 2019. https://doi.org/10.1093/ trstmh/trzO44 (Epub ahead of print).

4. Rayis DA, Ahmed MA, Omer EM, Adam I. Plasmodium vivax malaria among pregnant women in Eastern Sudan. Asian Pacific J Trop Dis. 2016;6:2021-3.

5. Abdallah TM, Abdeen MT, Ahmed IS, Hamdan HZ, Magzoub M, Adam I. Severe Plasmodium falciparum and Plasmodium vivax malaria among adults at Kassala Hospital, eastern Sudan. Malar J. 2013;12:148.

6. El-Amin EO, Elbashir MI, Elamin OE, Mukhtar Y, Abdo H, Abdul-Rahman I, et al. The underlying aetiologies of coma in febrile Sudanese children. Trans R Soc Trop Med Hyg. 2013;107:307-12.

7. Snounou G, Viriyakosol S, Jarra W, Thaithong S, Brown KN. Identification of the four human malaria parasite species in field samples by the polymerase chain reaction and detection of a high prevalence of mixed infections. Mol Biochem Parasitol. 1993;58:283-92.

8. Mahgoub H, Gasim GI, Musa IR, Adam I. Severe vivax malaria among Sudanese children at New Halfa hospitals, Eastern Sudan. Parasit Vectors. 2012;5:154.

9. Baird JK. Evidence and implications of mortality associated with acute Plasmodium vivax malaria. Clin Microbiol Rev. 2013;26:36-57.

10. Battle KE, Karhunen MS, Bhatt S, Gething PW, Howes RE, Golding N, et al. Geographical variation in Plasmodium vivax relapse. Malar J. 2014;13:144.

\section{Publisher's Note}

Springer Nature remains neutral with regard to jurisdictional claims in published maps and institutional affiliations. 